\title{
Surface Dipole Formation and Lowering of the Work Function by Cs Adsorption on InP(100) Surface
}

\author{
Yun Sun, Zhi Liu, and Piero Pianetta \\ Stanford Synchrotron Radiation Lab, Stanford, CA
}

\begin{abstract}
:
The Cs adsorption on InP(100) surface is studied with Synchrotron Radiation Photoelectron Spectroscopy. The charge transfer from Cs to the InP substrate is observed from the Cs induced In 4d and P 2p components, and this charge transfer results in surface dipole formation and lowering of the work function. The Cs 4d intensity saturates at coverage of one monolayer $(\mathrm{ML})$. However, a break point is observed at $0.5 \mathrm{ML}$, which coincides with the achievement of the minimum work function. This break point is due to the different vertical placement of the first and the second half monolayer of Cs atoms. Based on this information, a simple bi-layer structure for the Cs layer is presented. This bi-layer structure is consistent with the behavior of the charge transfer from the Cs to the InP substrate at different Cs coverages. This, in turn, explains why the work function decreases to a minimum at $0.5 \mathrm{ML}$ of $\mathrm{Cs}$ and remains almost constant beyond this coverage. The depolarization of the surface dipoles is attributed to the saturation of charge transfer to the surface In atoms and the polarization of the Cs atoms in the second half monolayer induced by the positively charged Cs atoms in the first half monolayer.
\end{abstract}




\section{Introduction:}

Research on Cs adsorption on metals, oxides and semiconductors has been studied for many years. Especially, the research on III-V semiconductors has led to a variety of applications including Negative Electron Affinity (NEA) photocathodes because Cs can lower the work function of semiconductors considerably ${ }^{1-7}$. Besides practical applications, the NEA surface also offers a very good opportunity to investigate the low-lying conduction bands as well as electron transport properties ${ }^{2}$. Recently, the low work function on Cs covered surfaces enabled interesting discoveries of energy transfer from molecular vibrations to electron excitations ${ }^{3}$.

Within the studies of Cs adsorption on III-V semiconductors, Cs/GaAs has been the most widely studied system ${ }^{6,8-17}$. Cs/InP has received less attention and published work is concentrated on the $\operatorname{InP}(110)$ cleaved surfaces because such surfaces are fairly well-defined $^{18-20}$. Recently, $\mathrm{InP}(100)$ is gaining more importance for the next generation of night vision technology because it can be lattice matched to $\mathrm{Ga}_{\mathrm{x}} \mathrm{In}_{1-\mathrm{x}} \mathrm{As}_{\mathrm{y}} \mathrm{P}_{1-\mathrm{y}}$ band engineered materials, serving as the emission surface for the photocathode. This brings an imminent demand for a scientific understanding of the Cs/InP(100) interface, which serves as the basis for the study of activation of $\operatorname{InP}(100)$ by Cs and O to achieve NEA.

The observed lowering of the work function is normally explained by the formation of dipole layer on the surface $e^{4,5,6}$. Assuming a one to one correspondence between an individual dipole and a surface Cs atom, the Topping model $^{7}$ has been used to explain the dependence of the overall dipole strength on the Cs coverage. In this work, we will show that the experimentally observed charge transfer from the Cs to the InP 
substrate as well as the structure of the Cs layer provides an atomic picture for the dipole formations and depolarization.

In this study, we observed that the Cs $4 \mathrm{~d}$ core level intensity as a function of deposition time on the $\operatorname{InP}(100)$ surface saturates at one monolayer (ML) and a breakpoint at $0.5 \mathrm{ML}$ is also seen. Photoemission measurements taken at two different photon energies demonstrate that the Cs atoms in the second half monolayer are higher than those in the first half monolayer. The charge transfer from the Cs atoms to the InP substrate slows down with increased Cs coverage. The surface dipole buildup and the work function change as a function of Cs coverage are explained by the bi-layer structure of the Cs layer.

\section{Experimental:}

The InP(100) wafers used are $\mathrm{Zn}$ doped, p-type with a carrier concentration of $5 \times$ $10^{17} \mathrm{~cm}^{-3}$ manufactured by Wafer Technology, U.K.. The chemical cleaning process developed in our earlier work ${ }^{21}$ is used to obtain a clean starting $\operatorname{In} \mathrm{P}(100)$ surface. In this process, the sample is first etched in 4:1:100 $\mathrm{H}_{2} \mathrm{SO}_{4}: \mathrm{H}_{2} \mathrm{O}_{2}: \mathrm{H}_{2} \mathrm{O}$ solution for 2 minutes to remove the native oxide and grow 0.6 - 1 ML of chemical oxide, rinsed with DI water, and then etched in 1:1 $\mathrm{H}_{2} \mathrm{SO}_{4}: \mathrm{H}_{2} \mathrm{O}$ to remove the remaining oxide. After this step, the surface is hydrogen terminated on the surface P sites. The sample is then transferred into the photoemission chamber and heated to $330^{\circ} \mathrm{C}$ to remove the $\mathrm{H}$ termination, resulting in an In terminated clean InP surface.

The Cs deposition is done by evaporation from a SAES Getters (Italy) chromate source. The Cs getter is carefully out-gassed before use ${ }^{22}$. Photocurrent from the sample 
is generated by a He-Ne laser (632.8nm). The work function of the sample is measured from the low energy cut-off of the photoemission spectra. The lack of core level peak shift during the Cs deposition indicates that there is no band bending caused by Cs adsorption.

Experiments are performed at beam line 8-1 (photon energy range 30-190 eV) and beam line 8-2 (photon energy range 200-1300 eV) at the Stanford Synchrotron Radiation Laboratory (SSRL). The photon energy range of beam line 8-1 gives the best combination of surface sensitivity and energy resolution for the In $4 d\left(E_{b}=16.5 e V\right), P 2 p$ $\left(E_{b}=135 e V\right)$ and $C s 4 d\left(E_{b}=78 e V\right)$ core levels and the valence band which are measured at hv $=70,165,120$ and $70 \mathrm{eV}$, respectively. Beam Line 8-2 is used when photon energy higher than $200 \mathrm{eV}$ is required.

The photoemission spectra are collected with a PHI (model 10-360) spherical energy analyzer with a multi-channel detector. The spectra are fitted with Voigt functions, which are Gaussian broadened Lorentzian line shapes. The spin-orbit splitting is fixed at $0.86 \mathrm{eV}$ for $\mathrm{P} 2 \mathrm{p}, 0.855 \mathrm{eV}$ for In $4 \mathrm{~d}$ and $2.28 \mathrm{eV}$ for Cs $4 \mathrm{~d}$.

\section{Results and discussion:}

\subsection{Cs Adsorption and the Change of Work Function}

The Cs $4 \mathrm{~d}$ intensity at $\mathrm{h} v=120 \mathrm{eV}$ as a function of deposition time is plotted in figure 1(a). The intensity stops increasing at an exposure time of approximately $250 \mathrm{~s}$. It is well established in the literature that Cs adsorption on III-V semiconductor surfaces saturates at one monolayer coverage at room temperature ${ }^{14,18}$ because of very weak Cs-Cs 
attraction. At this one monolayer of Cs coverage, the P 2p intensity at hv $=165 \mathrm{eV}$ decreases to $48 \%$ of the value for clean surface and the In $4 \mathrm{~d}$ intensity at hv $=120 \mathrm{eV}$ deceases to $44 \%$. This attenuation by adsorbed Cs is similar to what was reported by Kendelewicz et al on the InP(110) surface $^{18}$. Using an effective electron escape depth of $7.5 \AA$ in $C s,{ }^{23}$ we estimate the Cs layer thickness to be approximately $6 \AA$, which is close to, but slightly larger than, a single physical layer of atomic Cs atoms.

A break point in the Cs $4 \mathrm{~d}$ intensity can be clearly seen in figure 1(a) when 0.5ML of Cs has been deposited. This is also the point where the work function reaches a minimum, as plotted in figure 1(a). After this point, the work function stays almost constant. This behavior is similar to what has been reported previously for Cs on $\operatorname{GaAs}(100)^{24}$, Cs on $\operatorname{InP}(110)^{19,20}$ and Na on $\mathrm{GaP}(110)^{25}$. As plotted in figure 1(b), the photocurrent reaches a maximum at the $0.5 \mathrm{ML}$ point, a direct consequence of the achievement of minimum work function.

The break point in the Cs $4 \mathrm{~d}$ intensity can not be explained simply by a change of the Cs sticking coefficient. We can see this by checking the Cs 4d intensity as a function of Cs coverage at hv $=350 \mathrm{eV}$ which has a longer electron escape depth than $120 \mathrm{eV}$. This result is plotted in figure 2 where we can see that the change of slope at 0.5 ML is less obvious compared with the data taken at $\mathrm{h} v=120 \mathrm{eV}$. A plausible explanation for this effect is the formation of a Cs bi-layer structure. The second half monolayer of Cs is located at a larger distance above the substrate surface than the first half monolayer. Therefore, it becomes clear that the intensity of first half monolayer would be attenuated by the second half monolayer. This attenuation creates the break point seen in the total Cs intensity. At the higher photon energy of $350 \mathrm{eV}$ (i.e. higher photoelectron kinetic 
energy) the attenuation factor is smaller due to the longer electron escape depth resulting in smaller slope change at $0.5 \mathrm{ML}$. The fact that Cs atoms within the same monolayer are not at the same distance above the substrate is not surprising because they are not necessarily adsorbed on identical sites. A similar bi-layer structure for a monolayer of Cs was also observed on the Cs/GaAs(110) surface by $\mathrm{STM}^{17}$. Since the Cs atoms in the second half monolayer are further away from the surface, they can not transfer charge to the InP substrate as effectively. That is why the work function reaches minimum at 0.5 ML of Cs, which is discussed in more details below.

\subsection{Charge transfer from Cs to the $\operatorname{InP(100)}$ substrate}

The P 2p and In 4d spectra at different Cs coverages are shown in figure 3(a) and (b), respectively. Careful examination of the In $4 \mathrm{~d}$ and P 2p core level line shapes shows that a tail on the higher kinetic energy side appears upon Cs deposition. The tail on the $\mathrm{P}$ 2p keeps growing as the Cs coverage increases but for In 4d, the line shape change after 0.3 ML of Cs is small. The numerical fitting for P 2p at a Cs coverage of 0, 0.25, 0.5 and $1 \mathrm{ML}$ is shown in figure 4. For the clean $\operatorname{InP}(100)$ surface, only one component is needed to fit the $\mathrm{P} 2 \mathrm{p}$ spectrum ${ }^{21}$. When Cs is deposited on the surface, an additional component on the high kinetic energy side must be added to fit the peak. This component has a chemical shift of $0.47 \pm 0.04 \mathrm{eV}$ relative to the P $2 \mathrm{p}$ peak in bulk InP, indicating charge transfer from the adsorbed Cs atoms. This Cs induced component grows with the deposition of additional Cs while the chemical shift remains the same.

The fits for In $4 \mathrm{~d}$ at Cs coverages of 0, 0.1, 0.2 and $0.3 \mathrm{ML}$ are shown in figure 5. The In $4 \mathrm{~d}$ for the clean surface (0 ML Cs) is shown in figure 5 (a). It has a surface 
component with kinetic energy $0.47 \mathrm{eV}$ higher than that of the bulk peak ${ }^{21}$. When Cs is deposited on the surface, this component grows, indicating that the Cs induced component overlaps the surface component. Although we are not able to resolve the Csinduced component from the original surface shifted peak of the clean surface because of their very similar chemical shifts, we will still use the term "Cs induced component" to identify this peak for simplicity. This Cs induced component grows at first, but seems to be constant when the Cs coverage is higher than 0.3 ML. As in the case for the P 2p, the chemical shift of the Cs induced In $4 \mathrm{~d}$ component does not change during the Cs deposition.

The Cs induced components for the In $4 \mathrm{~d}$ and P 2p core levels indicate that some surface In and P atoms receive charge from the adsorbed Cs atoms. For simplicity, the notations of $\mathrm{In}(\mathrm{Cs})$ and $\mathrm{P}(\mathrm{Cs})$ are used to represent those atoms. From the ratio of the Cs induced peak to the bulk peak, the coverage of $\operatorname{In}(\mathrm{Cs})$ and $\mathrm{P}(\mathrm{Cs})$ atoms are estimated and plotted in figure 6, using the theoretical electron escape depth of $\operatorname{InP}^{26}$. We do not have to consider the attenuation caused by the Cs layer here, because this attenuation of the In(Cs) and the $\mathrm{P}(\mathrm{Cs})$ atoms and the attenuation of the bulk In and $\mathrm{P}$ atoms are the same and are canceled out in our calculation. The coverage of In(Cs) grows quickly at the early stages of Cs adsorption and reaches approximately $1 \mathrm{ML}$ when about $0.25 \mathrm{ML}$ of Cs is deposited. This implies that, at this stage, the Cs atoms do not have one-to-one chemical bonds with the surface In atoms. Thus it is not likely that the Cs atoms occupy top sites on In atoms when the Cs coverage is less than 0.25 ML. The most likely adsorption sites for the Cs atoms are hollow sites surrounded by four Indium atoms on the (100) surface. If one Cs atom occupies one such site, charge transfer will occur from the Cs atom to the 
adjacent In atoms. The In(Cs) coverage stays almost constant when the Cs coverage is more than 0.25 ML, indicating the saturation of the charge transfer from the adsorbed Cs atoms to the surface In atoms.

Unlike $\operatorname{In}(\mathrm{Cs})$, the $\mathrm{P}(\mathrm{Cs})$ coverage increases with Cs adsorption until a full layer of Cs is deposited, but the rate of increase becomes smaller with higher Cs coverage. The growth of the $\mathrm{P}(\mathrm{Cs})$ at the beginning is slower than the $\operatorname{In}(\mathrm{Cs})$ because the clean $\operatorname{In} \mathrm{P}(100)$ surface is In terminated. However, after the charge transfer from the Cs to the In has saturated, additional charge transfer from the Cs must go to the P. Therefore, the $\mathrm{P}(\mathrm{Cs})$ coverage continues to increase. The ultimate coverage of $\mathrm{P}(\mathrm{Cs})$ is about $1 \mathrm{ML}$, similar to In(Cs), indicating that the Cs can only transfer charge to the surface In and P atoms but not to In and P atoms in the layers below the surface.

\subsection{Surface Dipole and Depolarization}

The dependence of the InP work function on the Cs coverage can be explained conceptually by the formation of surface dipoles and dipole depolarization as a result of dipole-dipole repulsions ${ }^{7}$. However, we need to go beyond the conceptual explanation and try to understand this work function dependence at the atomic level. A side view of the $\mathrm{Cs} / \mathrm{InP}(100)$ interface is shown schematically in figure 7 . All the atoms are drawn to scale according to the assumptions given below. Indium atoms have a diameter of $2.81 \AA$ and P atoms have a diameter of $2.26 \AA$ when they are in an InP single crystal. We can only estimate the sizes of the Cs atoms on the $\operatorname{InP}(100)$ surface because we do not know their exact charge state. A Cs atom has a atomic radius of $2.6 \AA$, and an ionic radius between $1.81 \AA$ and $1.88 \AA$, depending on the coordination ${ }^{27}$. We know that the charge 
donation from the Cs to the InP substrate is partial, i.e., the Cs atoms are only partially ionic, so the covalent radius of $2.25 \AA$ is probably the best approximation for the Cs atoms in the first half monolayer. This is also very close to the average of the atomic and ionic radius, so we believe that it is a reasonable approximation. The atomic radius is used for the Cs atoms in the second half monolayer because they only transfer a very small amount of charge to the substrate, as shown in figure 6 . The actual size and location of the surface atoms, which require more careful studies, may differ from this drawing. However, the bi-layer structure of the Cs layer, on which our discussion is mainly based, will not be affected

In section 3.1, we attributed the break point in figure 1(a) to the bi-layer structure, with Cs atoms in the second half monolayer being higher above the substrate than those in the first half monolayer. We can learn why this is the case by examining the structure shown in figure 7. On an $\operatorname{InP(100)}$ surface, the distance between two adjacent In atoms, which represents the size of the surface unit cell, is only $4.15 \AA$. This is smaller than the estimated Cs diameter of $4.5 \AA$, which means that it is impossible to form one monolayer of Cs at the same vertical level on InP. Even if the actual diameter of Cs atoms were smaller than our estimate so that there would be just enough space for all of them to fit on the surface, this will force the positively charged Cs atoms to pack very close to each other. Without negatively charged atoms in between to hold them together, such as in ionic crystals, mutual repulsion will make such surface highly unstable and will cause the rearrangement of the Cs layer. Some of the Cs atoms will be pushed up, resulting in the bi-layer structure, as depicted in figure 7. 
With this model of surface structure in mind, we will see how the Cs adsorption is correlated with the change of the surface dipole layer and the lowering of the work function. Before 0.25 ML of Cs coverage is reached, the rapid lowering of the work function is caused by the large charge transfer from the adsorbed Cs atoms to the substrate, which builds a surface dipole layer. Between 0.25 ML and 0.5 ML of Cs coverage, Cs atoms continue to occupy the available adsorption sites. However, since the charge transfer to the surface In atoms has already saturated, further charge transfer can only go to the surface $\mathrm{P}$ atoms, which are further away from the adsorbed Cs atoms. As a result, this charge transfer is much smaller than the initial charge transfer from Cs to In, leading to less dipole strength being built in this stage. Therefore, the change of work function is smaller.

Between 0.5 ML and 1ML, Cs atoms are adsorbed above the first half monolayer of Cs. They are further away from the InP surface, so only a very small amount of charge is transferred to the substrate. However, this limited charge transfer does not cause further reduction of the work function. We postulate that it is because of an induced polarization of the Cs atoms in the second half monolayer. Those Cs atoms are almost neutral, with a large radius and a loosely bound 6s electron which is highly polarizable. They are easily polarized by their nearest neighbors - the positively charged Cs atoms in the first half monolayer. This induced polarization causes a slight separation of positive and negative charge in the Cs atoms in the second half monolayer and is the very reason for the attraction between the two half monolayers. The induced polarization of the Cs atoms in the second half monolayer builds two additional dipoles D2 and D3, as depicted in figure 7. The combined effect of D2 and D3 cancels out the additional dipole strength 
built by the small charge transfer from the Cs atoms in the second half monolayer to the InP substrate. Therefore, the work function does not change significantly when more than 0.5 ML of Cs is deposited even though there is additional charge transfer to the substrate at this stage.

As discussed above, our understanding of the Cs/InP(100) interface explains the dependence of the work function on the Cs coverage. At the same time, it provides the atomic origin for the depolarization effect of the surface dipoles when the Cs coverage is high: the saturation of the charge transfer and the induced polarization of the Cs atoms in the second half monolayer.

\section{Conclusions:}

The Cs/InP(100) interface formation at room temperature is studied. Cs coverage saturates on $\operatorname{InP}(100)$ surface at $1 \mathrm{ML}$ with a bi-layer structure. Cs induced components for both In 4d and P 2p core levels indicate charge transfer from the adsorbed Cs atoms to the InP substrate. The change of the charge transfer as a function of Cs coverage, together with the bi-layer nature of the Cs layer, helps us to explain the change of the work function as a function of Cs coverage. The initial quick decrease of the work function is caused by the large charge transfer from the Cs to the InP substrate, while the saturation of the Cs charge transfer to the surface In atoms causes the decrease of the work function to slow down between 0.25 ML and 0.5 ML of Cs coverage. Lastly, the lack of change in the work function between $0.5 \mathrm{ML}$ and $1 \mathrm{ML}$ of Cs coverage is consistent with the polarization of the Cs atoms in the second half monolayer induced by the positively charged Cs atoms in the first half monolayer, which generates a dipole in a reverse 
direction to offset the limited additional charge transfer from the Cs atoms in the second half monolayer to the substrate.

\section{Acknowledgements:}

This research is funded by Army Contract DAAD19-02-1-0396. It is also supported by Department of Energy, Office of Basic Energy Sciences, Division of Chemical Sciences. We would like to thank the SSRL staff for their support. We also would like thank Dr. Juan Maldonado of Stanford University for useful discussions. 


\section{References}

$1 \quad$ R. L. Bell, Negative Electron Affinity Devices. (Clarenden Press, Oxford, 1973).

2 Z. Liu, F. Machuca, P. Pianetta, W.E. Spicer, and R.F.W. Pease, Applied Physics Letters 85 (9), 1541-1543 (2004).

3 J.D. White, J. Chen, D. Matsiev, D.J. Auerbach, and A.M. Wodtke, Nature 433 (7025), 503-505 (2005). W. Monch, Journal of Vacuum Science \& Technology B 4 (4), 1085-1090 (1986).

$5 \quad$ W. Monch, Reports on Progress in Physics 53 (3), 221-278 (1990).

$6 \quad$ F. Bechstedt and M. Scheffler, Surface Science Reports 18 (5/6), 145-198 (1993).

$7 \quad$ J. Topping, Proc. R. Soc. London A 114, 67 (1927).

$8 \quad$ H.J. Clemens, J. Von Wienskowski, and W. Monch, Surface Science 78 (3), 648666 (1978).

$9 \quad$ J. Derrien and F. Arnauddavitaya, Surface Science 65 (2), 668-686 (1977).

10 N.J. Dinardo, T.M. Wong, and E.W. Plummer, Physical Review Letters 65 (17), 2177-2180 (1990).

11 P.N. First, R.A. Dragoset, J.A. Stroscio, R.J. Celotta, and R.M. Feenstra, Journal of Vacuum Science \& Technology A 7 (4), 2868-2872 (1989).

12 Z. Gedik, S. Ciraci, and I.P. Batra, Physical Review B 47 (24), 16391-16394 (1993).

13 M. Kamaratos and E. Bauer, Journal of applied physics 70 (12), 7564-7572 (1991).

14 T. Kendelewicz, P. Soukiassian, M.H. Bakshi, Z. Hurych, I. Lindau, and W.E. Spicer, Physical Review B 38 (11), 7568-7575 (1988). 
S.E. Kulkova, D.V. Khanin, and A. Subashiev, Nuclear Instruments and Methods in Physics Research, Section A 536 (3), 295-301 (2005).

16

21
T.J. Lee, B.H. Blott, and B.J. Hopkins, Journal of Physics F (Metal Physics) 1 (3), 309-319 (1971).

L.J. Whitman, J.A. Stroscio, R.A. Dragoset, and R.J. Celotta, Physical Review Letters 66 (10), 1338-1341 (1991).

T. Kendelewicz, P. Soukiassian, M.H. Bakshi, Z. Hurych, I. Lindau, and W.E. Spicer, Journal of Vacuum Science \& Technology B 6 (4), 1331-1335 (1988).

Th. Chasse, G. Neuhold, J.J. Paggel, and K. Horn, Surface Science 331/333 (pt A), 528-533 (1995).

L. Zhong, S. Gao, and D. Wang, Physical Review B 47 (15), 9791-9796 (1993).

Y. Sun, Z. Liu, F. Machuca, P. Pianetta, and W.E. Spicer, Journal of Applied Physics 97 (12), 1-7 (2005).

P. Pianetta, in Speciman Handling, Preparation, and Treatments in Surface Characterization, edited by Czanderna et al. (Kluwer Academic / Plenum Publishers, New York, 1998).

S. Tanuma, C.J. Powell, and D.R. Penn, Surface and Interface Analysis 21 (3), 165-176 (1994).

K. Yamada, J. Asanari, M. Naitoh, and S. Nishigaki, Surface Science 402-404 (13), 683-686 (1998).

D.A. Evans, G.J. Lapeyre, and K. Horn, Physical Review B 48 (3), 1939-1942 (1993). 
S. Tanuma, C.J. Powell, and D.R. Penn, Surface and Interface Analysis 17 (13), 927-939 (1991).

27 A.M. James and M.P. Loard, Macmillan's Chemical and Physical Data.

(Macmillan, London, UK, 1992). 


\section{Figure Captions:}

Figure 1. (a) Cs 4d Intensity at hv $=120 \mathrm{eV}$ and work function change at different Cs coverage (Cs intensity: •, work function: $\square$ ) (b) Photo current from InP(100) during Cs deposition (Cs intensity: •, photocurrent: curve) at different Cs coverage.

Figure 2. Cs 4d intensity as a function of Cs coverage at two different photon energies (hv $=120 \mathrm{eV}: \bullet, \mathrm{hv}=350 \mathrm{eV}: \square)$

Figure 3. (a). P2p (b) In 4d spectra at different Cs coverage. A component at higher kinetic energy side grows with more Cs deposited. Spectra are normalized to the same height to show the line shape change.

Figure 4. Fit for P 2p at different Cs coverage. (a) $0 \mathrm{ML}$, (b) $0.25 \mathrm{ML}$, (c) $0.5 \mathrm{ML}$, (d) 1 ML. Dots are experimental data and lines are the fitting result.

Figure 5. Fit for In 4d at different Cs coverage. (a) $0 \mathrm{ML}$, (b) $0.1 \mathrm{ML}$, (c) $0.2 \mathrm{ML}$, (d) 0.3 ML. Dots are experimental data and lines are the fitting result.

Figure 6. Coverage of $\operatorname{In}(\mathrm{Cs})$ and $\mathrm{P}(\mathrm{Cs})$ at different Cs coverage $(\mathrm{P}(\mathrm{Cs}): \bullet, \operatorname{In}(\mathrm{Cs}): \Delta)$.

Figure 7. Schematic side view of the Cs atoms on $\operatorname{InP}(100)$ substrate. All atoms are drawn to scale with their assumed sizes. The covalence diameter (d1) is used for the Cs atoms in the first half monolayer while the atomic diameter (d2) is used for the Cs atoms 
in the second half monolayer because they donate little charge to the substrate. D1 is the dipole between the substrate and the first half monolayer of Cs, D2 is the dipole between the first and second half monolayer of Cs and D3 is the dipole caused by the induced polarization of the second half monolayer of Cs. 


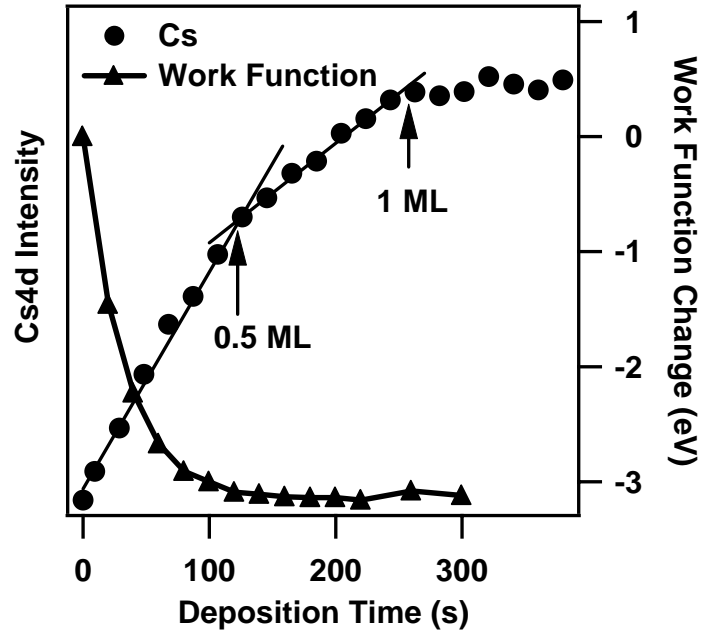

(a)

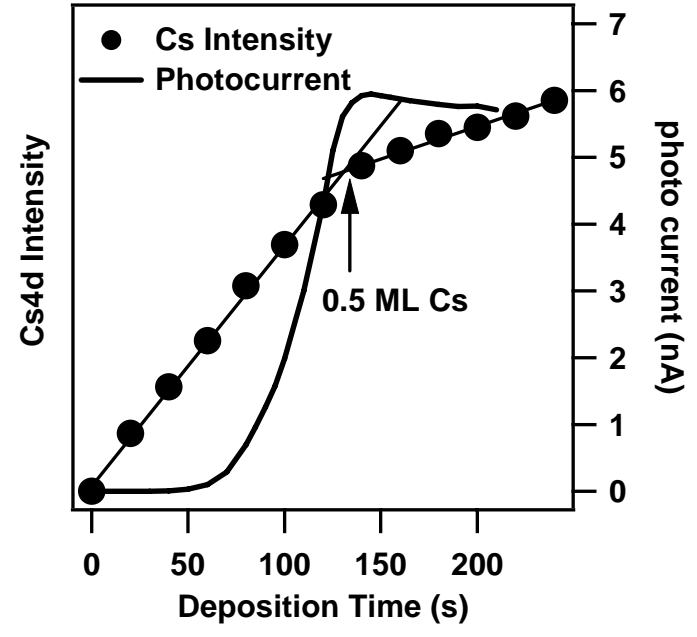

(b)

Figure 1

Y. Sun, et al. JVST A 


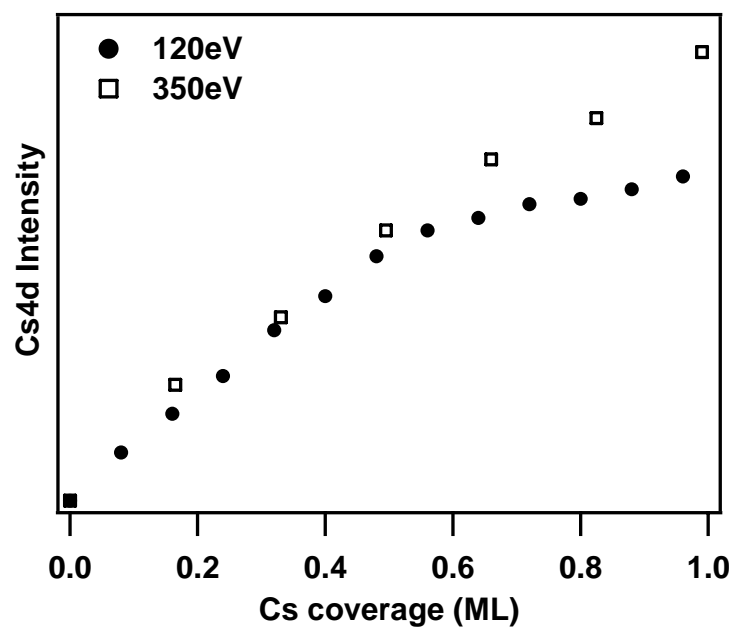

Figure 2

Y. Sun, et al. JVST A 

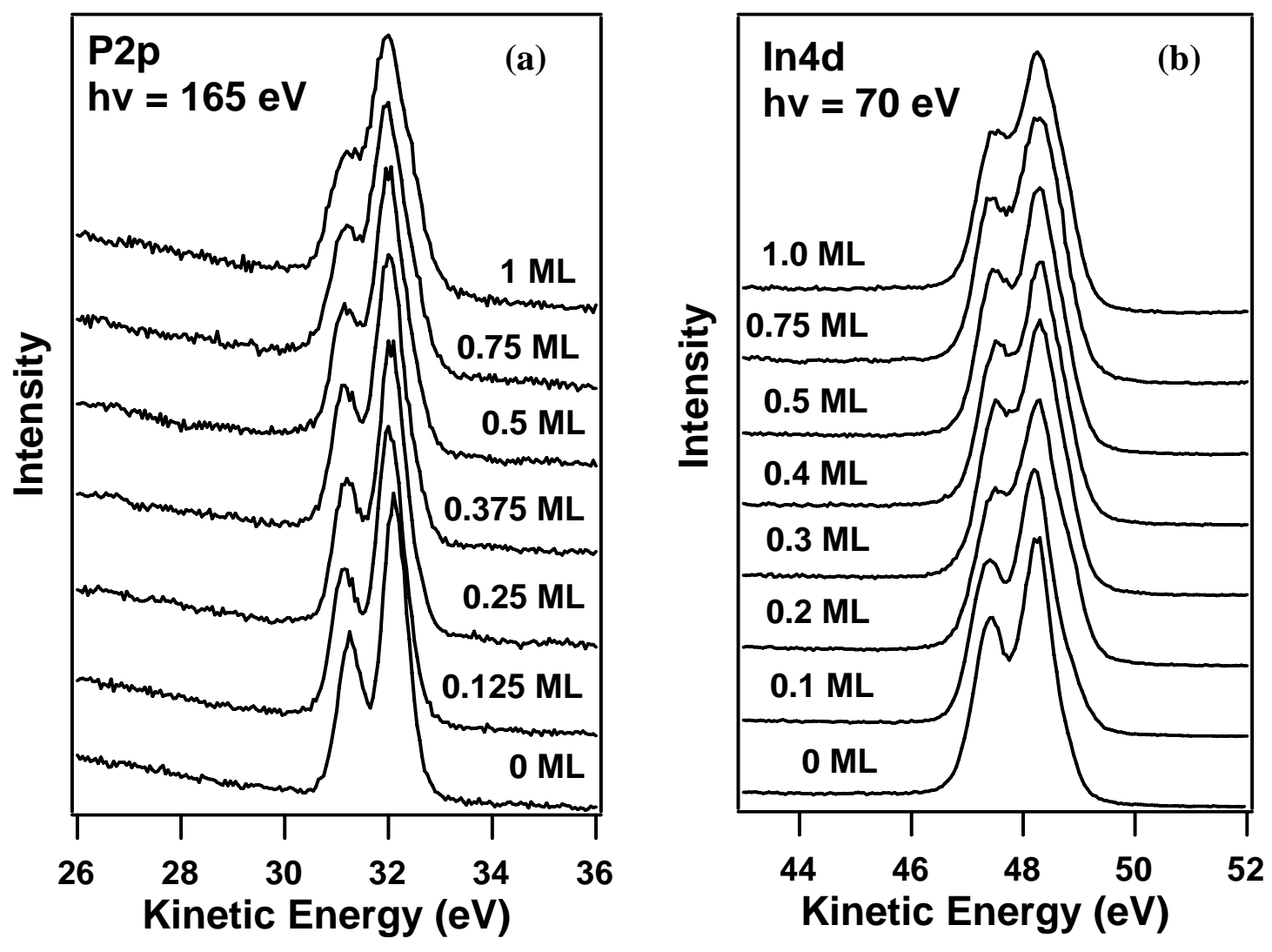

Figure 3

Y. Sun, et al. JVST A 

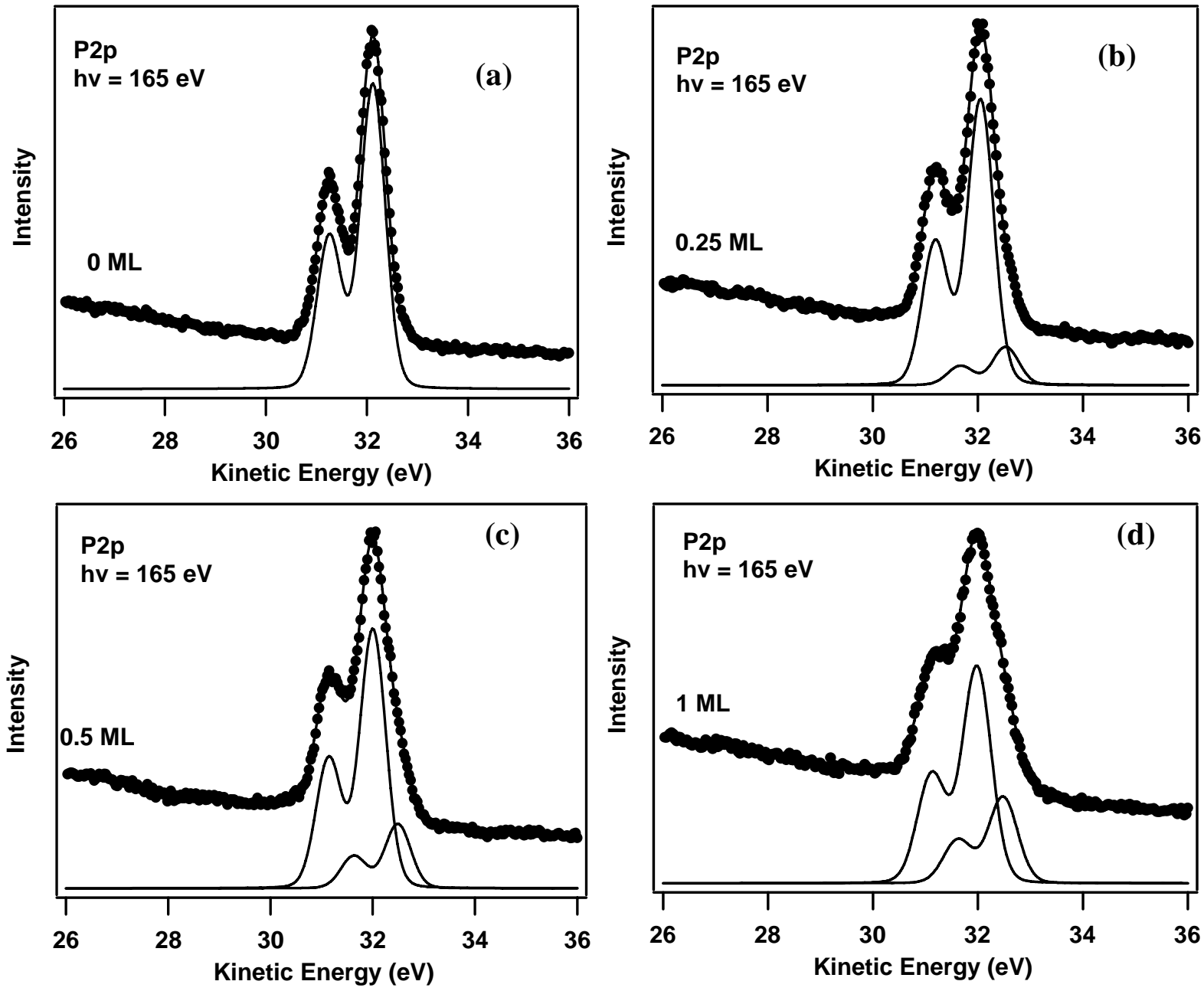

Figure 4

Y. Sun, et al. JVST A 

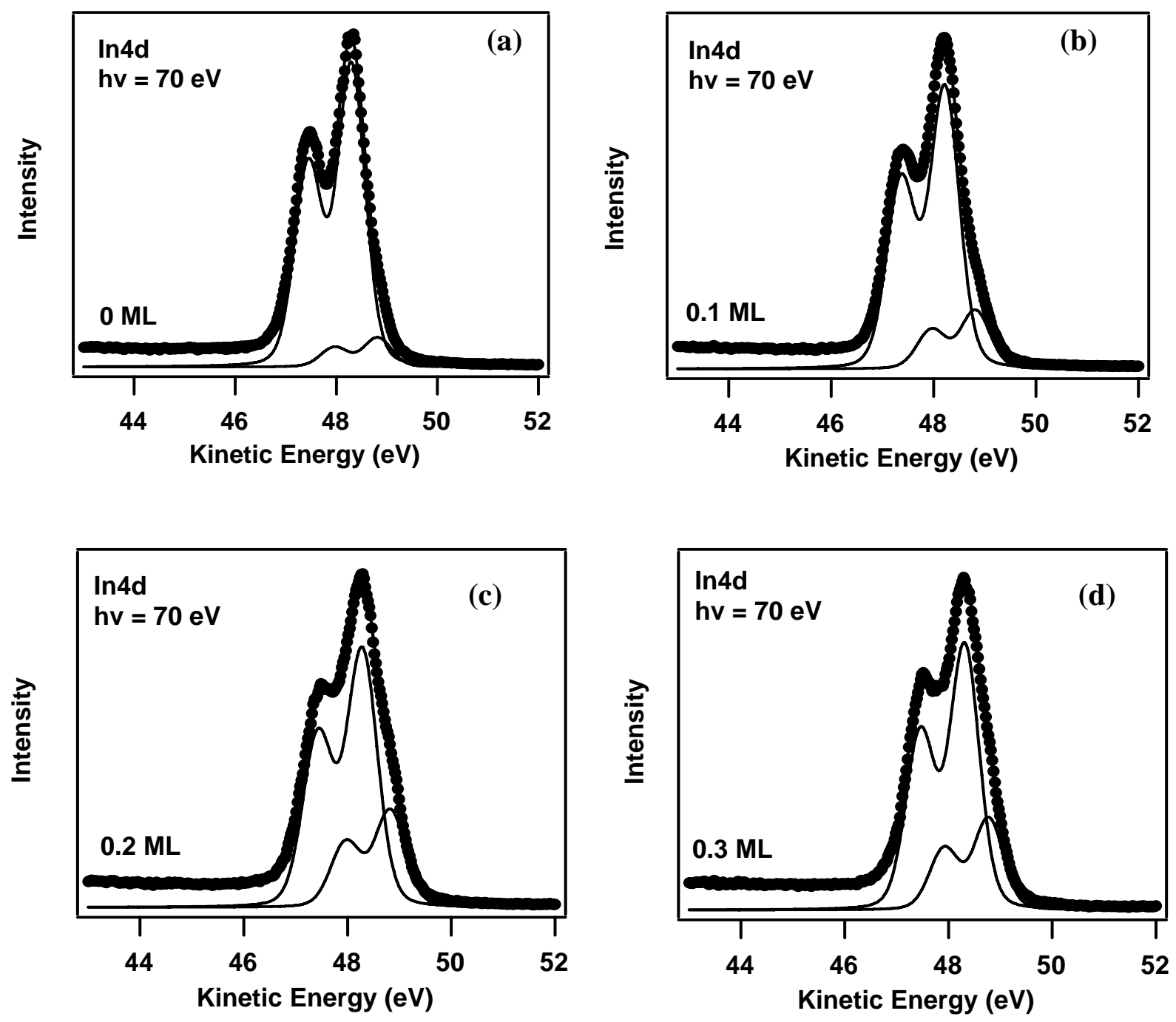

Figure 5

Y. Sun, et al. JVST A 


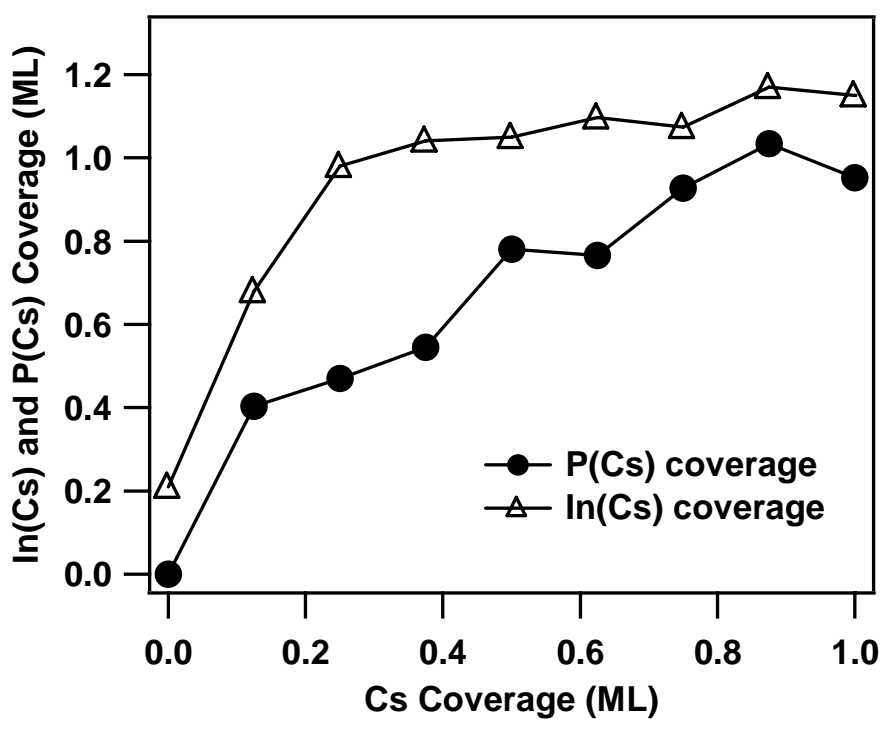

Figure 6

Y. Sun, et al. JVST A 

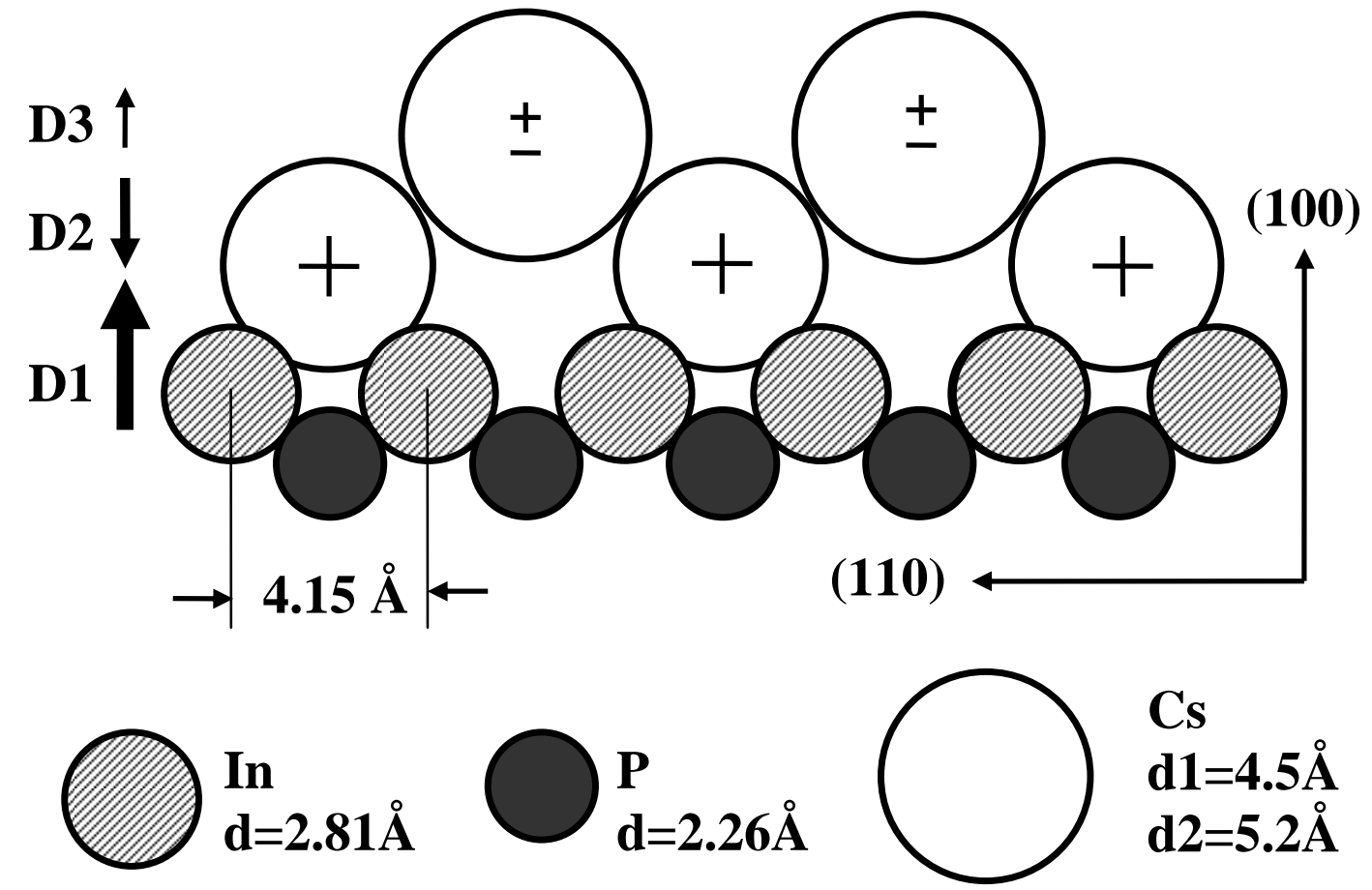

Figure 7

Y. Sun, et al. JVST A 